\title{
Special issue on DTIP 2014
}

\author{
Romolo Marcelli
}

Published online: 21 February 2015

(C) Springer Science+Business Media New York 2015

This Special Issue is composed by seven papers, selected and expanded from the contributions presented at the 2014 DTIP Symposium on MEMS and MOEMS (Design, Test, Integration \& Packaging of MEMS/MOEMS), held in Cannes, Cote d'Azur on 1-4 April 2014. It was a successful experience in the now long history of the DTIP Symposium, chaired by Bernard COURTOIS, CMP, Grenoble, France, with the significant contribution of the Vice General Chair Pascal NOUET, LIRMM, Montpellier, France and the Co-Chair Jean Michel KARAM, MEMSCAP, Bernin, France. This edition of the Symposium ended with the passing of the baton between Bernard Courtois, who diligently and with great efficiency has played the role of organizer of the event since 1999, and Pascal Nouet, which will continue in the role of Chair from 2015 onwards. So far, first of all the Editor conveys special thanks to Bernard for his continuous and valuable efforts for the past years and good luck to Pascal for the future and successful organization of the DTIP Symposium. Last, but not least, I would like to thank Prof. Yoshio Mita, from the University of Tokyo, Japan, for helping me as a Co-Chair in the CAD conference organization.

Technical areas covered by the CAD Conference of DTIP 2014, from which the papers belonging to this special issue have been selected, span from DC to RF applications including technological efforts, mechanical nonlinear properties and nano-characterization of the exploited device configurations. In detail:

\section{R. Marcelli $(\bowtie)$}

Institute for Microelectronics and Microsystems (CNR-IMM

Roma), National Research Council of Italy, Via del Fosso del Cavaliere, 10000133 Rome, Italy

e-mail: Romolo.Marcelli@cnr.it
The first paper: "Real-Time Characterization of Dielectric Charging in Contactless Capacitive MEMS", by Manuel Dominguez-Pumar et al., presents a new method to characterize the dynamics of the charge trapped in the dielectric layer of contactless microelectromechanical systems (MEMS). For sampled-time systems, this allows knowing the state of the net charge at each sampling time without distorting the measurement.

The second paper "Analysis of the adhesion effect in RF-MEMS switches using atomic force microscope", by Corina Birleanu and Marius Pustan, is an investigation of the adhesion effect on the mechanical response of MEMS components for RF switch application by using an atomic force microscope (AFM). The occurrence and strength of adhesion effects in MEMS devices depend on a variety of environmental, geometrical and operational parameters, carefully discussed in this contribution.

The third paper "Resonant Behavior Study of PZT Sensor Partially Immersed in Liquid Using PSO Method: Modeling and Experiment", by Mahnaz Shamshirsaz and Mohammad Maroufi, is an analysis of resonant piezoelectric-excited millimeter-sized cantilevers (PEMCs) in applications such as liquid level and density sensing. In this work, a PEMC is designed and fabricated to be used as a liquid level sensing platform. An analytical model is developed and applied to evaluate the behavior of this device with respect to different tip immersion depths.

The fourth paper "Analysis and Design of an Electrostatic MEMS Microphone using the PolyMUMPs Process", by Owen Casha et al., is the analysis and design of an electrostatic MEMS microphone using the PolyMUMPs process with an additional back-etch processing step. Circular and square (simply supported and clamped) diaphragm designs are considered and analyzed, with the 
final design being based on the clamped square diaphragm with a bottom sound port.

The fifth paper "Design, Microfabrication and Analysis of Polysilicon Thin Layers for MEMS Vibrating Structures", by Rodica Cristina Voicu et al., is a study of natural frequencies of an array of microcantilevers designed with different dimensions, analysed using computer simulations. Four different types of polysilicon were obtained and topography scan measurements were carried out using AFM in order to characterize the material properties. Mechanical properties such as Young's modulus have been investigated using the nanoindenter technique. The microcantilevers have been manufactured using surface micromachining technique with the structural material undoped and doped polysilicon.

The sixth paper "Nonlinear regime operation for a high resolution vibrating beam UGS seismometer", by Raphael Levy et al., discusses about MEMS vibrating beam accelerometers (VBAs), which use the variation of frequency of a vibrating beam attached to a proof mass to measure its applied acceleration. Considering inertial navigation applications, the beam is actuated in the linear regime to keep high Eigen frequency stability. Concerning the new application of MEMS accelerometers as UGS seismometers, the bias stability is not important anymore and the important parameter is the resolution at integration time of $1 \mathrm{~ms}$. It becomes then interesting to actuate the beam in the nonlinear regime. This paper presents a behavioral model of the vibrating beam accelerometer including the nonlinear terms.

The seventh paper "Nanomechanical and nanotribological characterization of a MEMS micromembrame supported by two folded hinges", by Marius Pustan et al., is an experimental investigation, with analytical and numerical studies, of a micromembrane supported by two folded hinges, which can be used as a flexible component in RF-MEMS switches or in optical applications. The experimental tests are performed to determine the micromembrane behavior under a mechanical loading. Mechanical properties under interest like the bending stiffness and stress have been studied.

I would like to thank all the authors who contributed to this special issue, hoping that the reader will enjoy the nice papers selected.

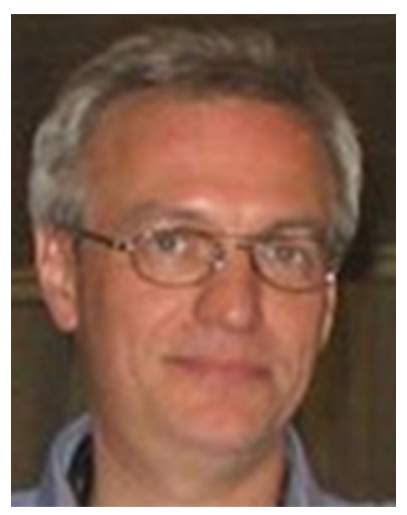

Romolo Marcelli was born in Roma, Italy, on February 26, 1958. He obtained the degree in Physics at the University of Roma "La Sapienza" in 1983. From 1987 he is with the National Research Council (CNR) of Italy, and from 1998 with the Institute for Microelectronics and Microsystems (IMM) of CNR. Presently, he is a Senior Researcher and Responsible of the Research Line on High Frequency Microsystems: Technologies and Reliability for Ground and Space Applications at CNR-IMM Roma. He was also Responsible Delegate of the IMM Director from October 2006 to May 2014. He is member of the IEEE since 1995, and of the IEEE Magnetism and Microwave Theory and Techniques Societies. He is also member of the American Physical Society and was member of the Administrative Committee for the IEEE Magnetics Society from 1995 to 1997. His past and current interests include technologies, design and test activities in Microwave Magnetics and RF MEMS. Currently, he is also involved in Microwave Imaging and Metamaterials at Microwave and Millimeter Wave Frequencies. In this framework, he also managed several national and international industrial and academic contracts, and he served as organizer and committee member in workshops and conferences. He is Referee for several journals in the fields of interest. He is Editor of two books on the linear and non-linear properties of magnetic microwave devices, and co-author of more than two hundred international papers and conference contributions. 